\title{
Field tensor network states
}

\author{
Anne E. B. Nielsen, ${ }^{1,2}$ Benedikt Herwerth, ${ }^{3}$ J. Ignacio Cirac, ${ }^{3,4}$ and Germán Sierra ${ }^{5}$ \\ ${ }^{1}$ Max-Planck-Institut für Physik komplexer Systeme, Nöthnitzer Strasse 38, D-01187 Dresden, Germany \\ ${ }^{2}$ Department of Physics and Astronomy, Aarhus University, DK-8000 Aarhus C, Denmark \\ ${ }^{3}$ Max-Planck-Institut für Quantenoptik, Hans-Kopfermann-Strasse 1, D-85748 Garching, Germany \\ ${ }^{4}$ Munich Center for Quantum Science and Technology (MCQST), Schellingstrasse 4, D-80799 München, Germany \\ ${ }^{5}$ Instituto de Física Teórica UAM/CSIC, Universidad Autónoma de Madrid, Cantoblanco, Madrid, Spain
}

(Received 6 February 2020; revised 24 November 2020; accepted 31 March 2021; published 16 April 2021)

\begin{abstract}
We define a class of tensor network states for spin systems where the individual tensors are functionals of fields. The construction is based on the path-integral representation of correlators of operators in quantum field theory. These tensor network states are infinite-dimensional versions of matrix product states and projected entangled pair states. We find the field tensor that generates the Haldane-Shastry wave function and extend it to two dimensions. We give evidence that the latter underlies the topological chiral state described by the Kalmeyer-Laughlin wave function.
\end{abstract}

DOI: 10.1103/PhysRevB.103.155130

\section{INTRODUCTION}

Tensor networks (TNs) are becoming a key tool to describe many-body quantum systems [1]. On the one hand, they can efficiently approximate quantum states of local Hamiltonians in thermal equilibrium, which has led to powerful numerical algorithms with applications in condensed matter and, to some extent, in high-energy physics [2]. On the other hand, they provide us with paradigmatic examples of strongly correlated states and thus allow us to investigate intriguing many-body quantum phenomena. For instance, they offer us a guide to classify symmetry-protected topological phases $[3,4]$, or to understand a large variety of topologically ordered behavior. In fact, states (or models) like the Affleck-KennedyLieb-Tasaki (AKLT) [5], string-net states [6], or resonating valence-bond states have a very simple description in terms of TNs. By simple we mean they have a small bond dimension, $D$, which limits the number of coefficients describing the tensors generating the many-body states. The description of such states in terms of TNs automatically opens up the possibility of using powerful tools in order to describe their physical properties by just inspecting a simple tensor. In one dimension, one can easily describe symmetries and string order parameters [7,8], or even gauge symmetries [9]. In two dimensions, apart from obtaining the physical symmetries, one can directly identify the topological properties or type of anyon excitations of the parent Hamiltonian [10].

Published by the American Physical Society under the terms of the Creative Commons Attribution 4.0 International license. Further distribution of this work must maintain attribution to the author(s) and the published article's title, journal citation, and DOI. Open access publication funded by the Max Planck Society.
There exist, however, some classes of states for which no exact expressions in terms of tensor network states of finite bond dimensions exist. Two prominent examples are critical states [11], and chiral topological states of gapped Hamiltonians in one- (1D) and two-dimensional (2D) spin lattices, respectively $[12,13]$. The reason behind the lack of description as a TN for the first stems from the fact that critical states violate the area law [14,15]. Specifically, the entanglement entropy of a connected region containing $L$ spins scales as $\propto \ln (L)[16,17]$, whereas for a matrix product state (MPS), the one-dimensional version of tensor network states, it is bounded by $2 \ln (D)$; therefore, in the thermodynamic limit for any finite $D$, there always exists some $L$ for which an MPS cannot cope with the amount of entanglement and thus it is impossible that it describes a critical state. The reason for the second class is more subtle and yet not fully understood; however, there are good reasons to believe that there exist obstructions due to the nonexistence of local Wannier states [18] (see, however, [19]). In fact, for Gaussian fermionic states, it is not possible to describe gapped chiral topological insulators [20]. ${ }^{1}$ We emphasize that here we mean an exact description; in fact, both classes of states may well be approximated efficiently with an error that decreases as $D$ increases [21-23].

The arguments above do not prevent the existence of exact descriptions of critical or chiral topological states with TNs of infinite bond dimensions. In [24], it was noted that the conformal field theory (CFT) formulation [25] of the HaldaneShastry state has similarities with MPS, and in [21-23], the CFT formulation was used to obtain MPS with a discrete, infinite bond dimension describing chiral topological states

\footnotetext{
${ }^{1}$ Even though those do not possess topological order, but rather belong to a symmetry-protected topological phase, they constitute a strong evidence for the impossibility of describing chiral topological phases corresponding to gapped local Hamiltonians.
} 
in two dimensions. From the tensor network perspective it is, however, desirable to use projected entangled pair states (PEPSs) to deal with 2D systems. Furthermore, although the approach of [21-23] can, in principle, be used to describe critical states in one dimension with open boundary conditions, it is more appropriate to use periodic boundary conditions for translationally invariant systems.

Here, we define field tensor networks (FTNs) for spin lattices in any dimension, where the bonds in the tensors are functions, the corresponding contractions are accomplished by a path integration, and the tensors themselves are functionals. The virtual space is hence continuous. We show how this approach can be used to describe translationally invariant critical systems, as well as the analogs of PEPSs for two-dimensional systems. Our construction is reminiscent of recent proposals for constructing tensor networks for quantum fields, where path integration is also employed [26,27]. In our case, however, we deal with discrete spin lattices and the construction is quite different. We give a procedure to compute the FTN for states whose coefficients in the spin basis can be written as vacuum correlators of a quantum field theory with a local action. In particular, we give an explicit construction for free boson CFTs and vertex operators. We also take advantage of the fact that the Haldane-Shastry state $[28,29]$, a prominent critical state, can be expressed in that form [24,30] to compute a FTN generating that state. The description allows both periodic and open boundary conditions. We also propose a FTN in two dimensions and give strong evidence that it represents a Kalmeyer-Laughlin state [12], a prototypical representative of chiral topological order.

\section{FTN IN ONE DIMENSION}

We consider a spin chain of $N$ spins of dimension $d$, and a translationally invariant state

$$
|\Psi\rangle=\sum_{s_{1}, \ldots, s_{N}=1}^{d} c_{s_{1}, \ldots, s_{N}}\left|s_{1}, \ldots, s_{N}\right\rangle .
$$

Let us start recalling the MPS, where

$$
c_{s_{1}, \ldots, s_{N}}=\sum_{n_{1}, \ldots, n_{N}=1}^{D} A_{n_{1}, n_{2}}^{s_{1}} \cdots A_{n_{N}, n_{1}}^{s_{N}},
$$

$D$ is the bond dimension, and for each value of $s=1, \ldots, d$, $A_{n, m}^{s}$ is a $D \times D$ matrix. In an analogous way we define (translationally invariant) field tensor network states (FTNSs) as

$$
c_{s_{1}, \ldots, s_{N}}=\int \mathcal{D}\left[\alpha_{1}\right] \cdots \mathcal{D}\left[\alpha_{N}\right] \mathcal{A}_{\alpha_{1}, \alpha_{2}}^{s_{1}} \cdots \mathcal{A}_{\alpha_{N}, \alpha_{1}}^{s_{N}} .
$$

Here, $\alpha_{n}: \mathbb{R} \rightarrow \mathbb{R}$ belong to the set of square-integrable functions and also include a constant function, and for each value of $s=1, \ldots, d, \mathcal{A}_{\alpha, \beta}^{s}$ are functionals of $\alpha, \beta$. Note that $\Psi$ has the same structure as a MPS, where the indices of the matrices are replaced by the functions $\alpha_{n}$, and the sum over repeated indices is replaced by a path integral.

We also define the functionals

$$
\mathcal{A}_{\alpha, \alpha^{\prime}}^{s_{1}, \ldots, s_{n}}=\int \mathcal{D}\left[\alpha_{2}\right] \cdots \mathcal{D}\left[\alpha_{n}\right] \mathcal{A}_{\alpha, \alpha_{2}}^{s_{1}} \cdots \mathcal{A}_{\alpha_{n}, \alpha^{\prime}}^{s_{n}}
$$

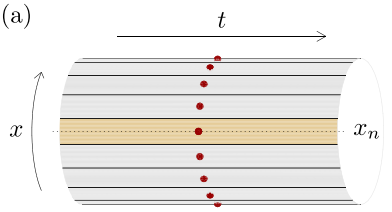

(c)

(b)

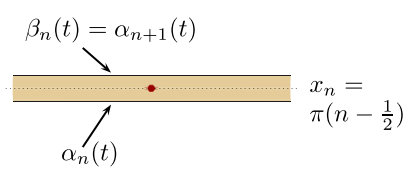

(d)

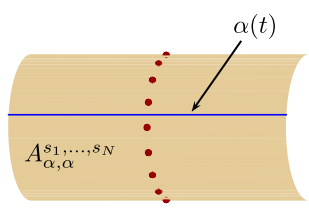

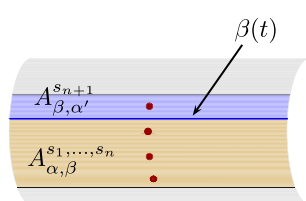

FIG. 1. (a) The CFT is defined on a cylinder of radial (axial) coordinate $x(t)$. The spins (represented as red points) are punctures at positions $\left(x_{n}, 0\right)$. (b) The path integral defining the state is extended over the cylinder. The functional $A_{\alpha_{n}, \alpha_{n+1}}^{s_{n}}$ corresponds to the integration over a stripe, where the boundary conditions for $\varphi$ are given by $\alpha_{n}$ and $\alpha_{n+1}$. Panels (c) and (d) illustrate the sewing and closing conditions, respectively.

fulfilling

$$
\begin{aligned}
\mathcal{A}_{\alpha, \alpha^{\prime}}^{s_{1}, \ldots, s_{n+1}} & =\int D[\beta] \mathcal{A}_{\alpha, \beta}^{s_{1}, \ldots, s_{n}} \mathcal{A}_{\beta, \alpha^{\prime}}^{s_{n+1}}, \\
c_{s_{1}, \ldots, s_{N}} & =\int D[\alpha] \mathcal{A}_{\alpha, \alpha}^{s_{1}, \ldots, s_{N}},
\end{aligned}
$$

which we will call "sewing" and "closing" conditions.

Example. Particularly interesting examples of FTNSs are those for which the coefficient can be written in terms of correlators of a simple CFT in $1+1$ dimensions and a local action. Specifically, we here study a family of critical states in one dimension with

$$
c_{s_{1}, \ldots, s_{N}} \propto \delta_{\sum_{n} s_{n}, 0} \prod_{n} \chi_{s_{n}} \prod_{n>m}\left\{\sin \left[\left(x_{n}-x_{m}\right) / N\right]\right\}^{2 q^{2} s_{n} s_{m}} .
$$

Here, $x_{n}=\pi\left(n-\frac{1}{2}\right), q$ is a real number, $\chi_{s_{n}}$ is a phase factor that may depend on $s_{n}$, and $s_{n}= \pm 1$. The member of the family with $q=1 / 2$ and $\chi_{s_{n}}=e^{i n \pi\left(s_{n}-1\right) / 2}$ is the ground state of the Haldane-Shastry model, which has been extensively studied in the literature as a paradigm of criticality. The wave function (6) has also been used as an ansatz for the ground state of the Hamiltonian of the XXZ spin-1/2 chain in the critical regime with anisotropy parameter $\Delta=-\cos \left(4 \pi q^{2}\right)$ [24].

The states (6) violate the area law, so that they cannot be written as MPSs with finite bond dimension. Nevertheless, we will show here how they can be expressed as FTNSs.

It is not difficult to show that [31]

$$
c_{s_{1}, \ldots, s_{N}} \propto\left\langle\chi_{s_{1}}: e^{i q s_{1} \varphi\left(\mathbf{r}_{1}\right)}: \cdots \chi_{s_{N}}: e^{i q s_{N} \varphi\left(\mathbf{r}_{N}\right)}:\right\rangle_{0},
$$

where $\varphi$ is a real scalar field defined on a cylinder of circumference $\pi N,::$ denotes normal ordering, the $\mathbf{r}_{n}=\left(x_{n}, 0\right)$ are points in cylindrical coordinates (see Fig. 1), and the expectation value is taken in the vacuum. In this case, using the path-integral representation we have

$$
c_{s_{1}, \ldots, S_{N}} \propto \int D[\varphi] e^{-S} e^{i q \sum_{n=1}^{N} s_{n} \varphi\left(\mathbf{r}_{n}\right)} \prod_{n} \chi_{s_{n}},
$$


where

$$
S=\frac{1}{8 \pi} \int_{0}^{\pi N} d x \int_{-\infty}^{\infty} d t\left\{\left[\partial_{x} \varphi(x, t)\right]^{2}+\left[\partial_{t} \varphi(x, t)\right]^{2}\right\}
$$

is the Euclidean action of the boson field. Notice that Eq. (9) vanishes if $\varphi$ is a constant $\varphi_{0}$ which upon integration generates the constraint $\sum_{n} s_{n}=0$ appearing in Eq. (6).

In order to find the FTNS representation of Eq. (7), we rewrite

$$
\int D[\varphi]=\int D\left[\alpha_{1}\right] \cdots D\left[\alpha_{N}\right] \int^{\prime} D\left[\varphi_{1}\right] \cdots \int^{\prime} D\left[\varphi_{N}\right] .
$$

Here, $\alpha_{n}(t)$ is a function of $t$ only, and $\varphi_{n}(x, t)$ is defined in the interval $(x, t) \in\left[x_{n}-\delta, x_{n}+\delta\right] \times \mathbb{R}$ with $\delta=\pi / 2$, and the ' indicates that it fulfills the boundary condition (see Fig. 1)

$$
\begin{aligned}
& \varphi_{n}\left(x_{n}-\delta, t\right)=\alpha_{n}(t), \\
& \varphi_{n}\left(x_{n}+\delta, t\right)=\alpha_{n+1}(t) .
\end{aligned}
$$

Thus, we simply identify

$$
\mathcal{A}_{\alpha, \alpha^{\prime}}^{s_{1}, \ldots, s_{L}}=\int^{\prime} D[\varphi] e^{-S} e^{i q \sum_{j=1}^{L} s_{j} \varphi\left(x_{j}, 0\right)} \prod_{n=1}^{L} \chi_{s_{n}},
$$

where the ' indicates that $\varphi$ has $\alpha=\alpha_{1}$ and $\alpha^{\prime}=\alpha_{L+1}$ as boundary conditions, and $S$ is defined as in Eq. (9) but with the integral in $x$ restricted to the interval $\left[x_{1}-\delta, x_{L}+\delta\right]$. Note that Eq. (3) trivially follows. The sewing and closing conditions (5) are represented for this case in Figs. 1(c) and $1(d)$.

In the Supplemental Material [32], using Green's function techniques, we explicitly compute

$$
\mathcal{A}_{\alpha, \alpha^{\prime}}^{s_{1}, \ldots, s_{L}} \propto e^{i \varphi_{0} q \sum_{j} s_{j}} e^{S_{L}^{(0)}+S_{L}^{(1)}+S_{L}^{(2)}} \prod_{n=1}^{L} \chi_{s_{n}},
$$

where

$$
\begin{gathered}
S_{L}^{(0)}=2 q^{2} \sum_{L \geqslant n>m \geqslant 1} s_{n} s_{m} \ln \frac{\sin \left[\left(x_{n}-x_{m}\right) /(2 L)\right]}{\sin \left[\left(x_{n}+x_{m}\right) /(2 L)\right]}, \\
S_{L}^{(1)}=\frac{1}{64 \pi^{2}} \int_{\mathbb{R}} d t d t^{\prime} \vec{\alpha}(t) U_{L}\left(t-t^{\prime}\right) \vec{\alpha}\left(t^{\prime}\right)^{T}, \\
S_{L}^{(2)}=\frac{q}{4 \pi} \int_{\mathbb{R}} d t \sum_{n=1}^{L} s_{n} \vec{\alpha}(t)\left(\vec{v}_{L, n}(t)^{T}-\vec{v}_{L, n}(t)^{\dagger}\right),
\end{gathered}
$$

and $\varphi_{0}$ is the zero mode of the boson field that has been subtracted from the functions $\alpha, \alpha^{\prime}$ to guarantee their normalizability. Here $\vec{\alpha}=\left(\alpha, \alpha^{\prime}\right)$, and $U$ is a $2 \times 2$ matrix with elements

$$
\begin{aligned}
& U_{L, 11 \text { or } 22}(t)=\frac{2}{L^{2}}\left[\frac{1}{\sinh ^{2}\left(\frac{t}{2 L}\right)}-\left(\frac{2 L}{t}\right)^{2}\right]-8 P^{\prime}\left(\frac{1}{t}\right), \\
& U_{L, 12 \text { or } 21}(t)=\frac{2}{L^{2} \cosh ^{2}\left(\frac{t}{2 L}\right)},
\end{aligned}
$$

and

$$
\vec{v}_{L, n}(t)=\frac{1}{L}\left(\operatorname{coth}\left(\frac{t-i x_{n}}{2 L}\right),-\tanh \left(\frac{t-i x_{n}}{2 L}\right)\right),
$$

where $P^{\prime}\left(\frac{1}{t}\right)=-\frac{1}{2}\left(\frac{1}{(t+i 0)^{2}}+\frac{1}{(t-i 0)^{2}}\right)$ is the derivative of the principal value distribution $P\left(\frac{1}{t}\right)$.

Chiral version. We aim at also being able to describe chiral states, and as a test case, we next consider a chiral formulation of the critical states (6). In this formulation the states are defined in terms of a chiral free boson field $\varphi(z)$, which depends on $z$, but not on its conjugate $\bar{z}$. The states are again given by Eq. (7), except that the vertex operators now take the form $: e^{i q s_{n} \varphi\left(z_{n}\right)}:$, where $q \in \mathbb{R}$ and $z_{n}=t+i x_{n}$ [the wave function obtained with these chiral vertex operators coincides with Eq. (6) except that $\sqrt{2} q$ is replaced by $q$ ]. This correlator can be written as in Eq. (8) with a chiral action [33] employed to study the edge excitations in the quantum Hall effect [34]. However, the slicing of the path integral into the intervals $(x, t) \in\left[x_{n}-\delta, x_{n}+\delta\right] \times \mathbb{R}$ introduces boundaries that mix the left- and right-moving modes of the bosonic field, which in turn complicates the approach.

We notice, however, that in Eq. (16) there are two parts related by complex conjugation. Moreover, Eq. (14) comes from a Green's function with four terms where only one of them is analytic in the location of the vertex operators. It is therefore natural to expect that one obtains the chiral state by selecting only one of those parts. We use this property to define the new tensors

$$
\hat{\mathcal{A}}_{\alpha, \alpha^{\prime}}^{s_{1}, \ldots, s_{L}}=e^{i \varphi_{0} q \sum_{j} s_{j}} L^{-\frac{1}{2} L q^{2}} e^{\hat{S}_{L}^{(0)}+S_{L}^{(1)}+\hat{S}_{L}^{(2)}} \prod_{n=1}^{L} \chi_{s_{n}},
$$

where

$$
\begin{gathered}
\hat{S}_{L}^{(0)}=q^{2} \sum_{L \geqslant n>m \geqslant 1} s_{n} s_{m} \ln \left[2 \sin \left(\frac{x_{n}-x_{m}}{2 L}\right)\right], \\
\hat{S}_{L}^{(2)}=\frac{q}{4 \pi} \int_{\mathbb{R}} d t \sum_{n=1}^{L} s_{n} \vec{\alpha}(t) \vec{v}_{L, n}(t)^{T} .
\end{gathered}
$$

The factor $L^{-\frac{1}{2} L q^{2}}$ guarantees that Eq. (19) satisfies the sewing condition (5).

The wave function that one obtains using Eq. (19) coincides with Eq. (6) (although with $q$ replaced by $\sqrt{2} q$, so that the Haldane-Shastry state now corresponds to $q=1 / \sqrt{2}$ ). This is not evident when comparing Eqs. (19)-(21) with Eqs. (13)-(16) as they look very different, and it is not obvious that they are related by a gauge transformation [1]. However, one can prove this statement by showing that Eq. (19) fulfills the sewing condition (5) and that by closing Eq. (5b) one indeed obtains the Haldane-Shastry wave function. We show that in the Supplemental Material [32], where we use the translational invariance of the action restricted to the strip along the $t$ coordinate, which allows us to diagonalize in $k$ space. Thus, the procedure leading to Eq. (19) provides a field theory version of the chiral vertex operator in CFT.

\section{FTN IN TWO DIMENSIONS}

The constructions presented above can be straightforwardly extended to represent states in two dimensions, corresponding to, e.g., a square spin lattice. We construct it on a cylinder, although one can similarly use a torus. The strip $\left[x_{n}-\delta, x_{n}+\delta\right] \times \mathbb{R}$ considered above is replaced by the rectangle $\left[x_{n}-\delta, x_{n}+\delta\right] \times\left[t_{m}-\delta^{\prime}, t_{m}+\delta^{\prime}\right]$. We define 
(a)
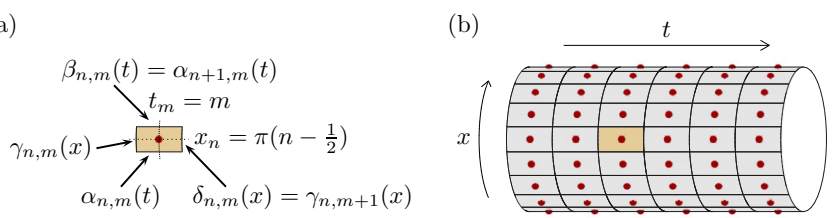

FIG. 2. FTNS in two dimensions: (a) functions on which $A_{\alpha_{n, m}, \beta_{n, m}, \gamma_{n, m}, \delta_{n, m}}^{s}$ depends upon, and (b) arrangement on a cylinder.

the functional $A_{\alpha_{n, m}, \beta_{n, m}, \gamma_{n, m}, \delta_{n, m}}^{s}$, which depends on the functions $\alpha_{n, m}, \beta_{n, m}:\left[t_{m}-\delta^{\prime}, t_{m}+\delta^{\prime}\right] \rightarrow \mathbb{R}$ and $\delta_{n, m}, \gamma_{n, m}:\left[x_{n}-\right.$ $\left.\delta, x_{n}+\delta\right] \rightarrow \mathbb{R}$ [see Fig. 2(a)]. By arranging the functionals along the cylinder [Fig. 2(b)], identifying functions like in Fig. 2(a), and integrating over them, one can construct states very much in the same way as one builds PEPSs in two dimensions.

Example. Again, an illustrative example is provided by states that are expressed in terms of a simple CFT with a local action. For instance, we can define a spin state as (see Fig. 2)

$$
A_{\alpha_{n, m}, \beta_{n, m}, \gamma_{n, m}, \delta_{n, m}}^{s}=\int^{\prime} D[\varphi] e^{-S[\varphi]} e^{i q s \varphi\left(x_{n}, t_{m}\right)} .
$$

Here, $\varphi:\left[x_{n}-\delta, x_{n}+\delta\right] \times\left[t_{m}-\delta^{\prime}, t_{m}+\delta^{\prime}\right] \rightarrow \mathbb{R}$ is a real, scalar field in $1+1$ dimensions, and with the boundary conditions

$$
\begin{aligned}
\varphi\left(x_{n}-\delta, t\right) & =\alpha_{n, m}(t), \\
\varphi\left(x_{n}+\delta, t\right) & =\beta_{n, m}(t), \\
\varphi\left(x, t_{m}-\delta^{\prime}\right) & =\gamma_{n, m}(x), \\
\varphi\left(x, t_{m}+\delta^{\prime}\right) & =\delta_{n, m}(x) .
\end{aligned}
$$

We then carry out the path integral in Eq. (22). The main technical tool to do this is to apply a conformal map that transforms the rectangle $\left[x_{n}-\delta, x_{n}+\delta\right] \times\left[t_{m}-\delta^{\prime}, t_{m}+\delta^{\prime}\right]$ into the complex upper-half plane in terms of Jacobi elliptic functions. In the case $(n, m)=(1,0)$ one finds (see the Supplemental Material [32])

$$
A_{\alpha, \beta, \gamma, \delta}^{s}=e^{S^{(1)}+S^{(2)}}
$$

where

$$
\begin{gathered}
S^{(1)}=\frac{1}{64 \pi^{2}} \int_{\mathcal{I}} d \xi d \xi^{\prime} \vec{\alpha}(\xi) U\left(\xi, \xi^{\prime}\right) \vec{\alpha}\left(\xi^{\prime}\right)^{T}, \\
S^{(2)}=\frac{q s}{4 \pi} \int_{\mathcal{I}} d \xi \vec{\alpha}(\xi)\left(\vec{v}(\xi)^{T}-\vec{v}(\xi)^{\dagger}\right),
\end{gathered}
$$

with $\vec{\alpha}=(\alpha, \beta, \gamma, \delta), \xi_{1}=\xi_{2}=t$, and $\xi_{3}=\xi_{4}=x$. The integration domain $\mathcal{I}$ is adapted to the type of variables involved. $U$ is a $4 \times 4$ matrix, some of whose elements are (the complete matrix is given in the Supplemental Material [32])

$$
\begin{aligned}
& U_{11}\left(t, t^{\prime}\right)=8\left(\frac{c n(t) d n(t) c n\left(t^{\prime}\right) d n\left(t^{\prime}\right)}{\left(\operatorname{sn}(t)-\operatorname{sn}\left(t^{\prime}\right)\right)^{2}}-\frac{1}{\left(t-t^{\prime}\right)^{2}}-P^{\prime} \frac{1}{t-t^{\prime}}\right), \\
& U_{33}\left(t, t^{\prime}\right)=8\left(\frac{k^{\prime 2} \widetilde{c n}(t) \widetilde{s n}(t) \widetilde{c n}\left(t^{\prime}\right) \tilde{s n}\left(t^{\prime}\right)}{\left(\widetilde{d n}(t)-\widetilde{d n}\left(t^{\prime}\right)\right)^{2}}-\frac{1}{\left(t-t^{\prime}\right)^{2}}-P^{\prime} \frac{1}{t-t^{\prime}}\right), \\
& U_{12}\left(t, t^{\prime}\right)=U_{21}\left(t, t^{\prime}\right)=8 k \frac{c n(t) d n(t) c n\left(t^{\prime}\right) d n\left(t^{\prime}\right)}{\left(1-k^{2} \operatorname{sn}(t) \operatorname{sn}\left(t^{\prime}\right)\right)^{2}}, \\
& U_{34}\left(t, t^{\prime}\right)=U_{43}\left(t, t^{\prime}\right)=8 k^{\prime 4} \frac{\widetilde{c n}(t) \widetilde{s n}(t) \widetilde{c n}\left(t^{\prime}\right) \tilde{s n}\left(t^{\prime}\right)}{\left(\widetilde{d n}(t)+\widetilde{d n}\left(t^{\prime}\right)\right)^{2}}, \\
& U_{22}\left(t, t^{\prime}\right)=U_{11}\left(t, t^{\prime}\right), \quad U_{44}\left(t, t^{\prime}\right)=U_{33}\left(t, t^{\prime}\right) .
\end{aligned}
$$

Here, $s n(t), c n(t)$, and $d n(t)$ are Jacobi elliptic functions of modulus $k$ and $\widetilde{s n}(t), \widetilde{c n}(t)$, and $\widetilde{d n}(t)$ of modulus $k^{\prime}=$ $\sqrt{1-k^{2}}$, where $k$ is determined from the aspect ratio $\delta / \delta^{\prime}$ of the considered rectangle [32]. In the limit $k \rightarrow 1$, the rectangle degenerates into a strip and we recover the matrix elements (17).

As in the previous example we can truncate this functional to a chiral one,

$$
\hat{A}_{\alpha, \beta, \gamma, \delta}^{s}=\chi_{s} e^{S^{(1)}+\hat{S}^{(2)},}
$$

where the phase factor $\chi_{s}$ can be chosen at will and

$$
\hat{S}^{(2)}=\frac{q s}{4 \pi} \int_{\mathcal{I}} d \xi \vec{\alpha}(\xi) \vec{v}(\xi)^{T} .
$$

We here consider the system on a cylinder, and to remove the virtual degrees of freedom on the boundaries, we take the rectangular regions for the boundary spins to go all the way to infinity. These boundary tensors can be obtained following the same approach as for the tensors in the bulk. We conjecture that sewing these amplitudes we get the wave function

$$
c_{s_{1}, \ldots, s_{N}} \propto \delta_{\sum_{n} s_{n}, 0} \prod_{n} \chi_{s_{n}} \prod_{n>m}\left(z_{n}-z_{m}\right)^{q^{2} s_{n} s_{m}}
$$

with $z_{n}=t_{n}+i x_{n}$. When $q=1 / \sqrt{2}$, Eq. (30) is a 2D topological state in the same universality class as the bosonic Laughlin state at filling fraction $1 / 2$, and the KalmeyerLaughlin wave function is obtained for $N \rightarrow \infty$ [35]

\section{CONCLUSIONS}

We introduced a class of TN constructed using functionals of fields that are contracted by means of the path integral of the functions defined on the links of the network. These tensors satisfy sewing and closing conditions that are similar to those 
employed in the construction of the scattering amplitudes in string theory $[36,37]$.

We illustrate our approach using a massless boson in two dimensions that allows us to derive the Haldane-Shastry wave function that describes a critical state in the universality class given by the WZW model $S U(2)_{1}$. We also conjecture the field tensor that generates the Kalmeyer-Laughlin state, which suggests that the chiral PEPSs underlying topological chiral states in two dimensions require infinite bond dimension. The latter suggestion could be further studied by truncating the field variables to a finite number of modes in which case the field tensor provides a PEPS with finite bond dimension. We have here focused on lattice states, but, utilizing the techniques in [38] to approach the continuum limit of the states, one could similarly describe continuum states. The definition of field tensor network states applies equally well to other types of lattices than those considered here. Our approach also allows a way to study topological chiral states based on the symmetry properties of the field tensors.

\section{ACKNOWLEDGMENTS}

We would like to thank A. Gasull, E. López, and A. Tilloy for conversations. J.I.C. is supported by the Deutsche Forschungsgemeinschaft (DFG, German Research Foundation) under Germany's Excellence Strategy EXC2111390814868 , and by the European Union through the ERC grant QUENOCOBA, ERC-2016-ADG (Grant No. 742102). G.S. is supported by the Ministerio de Ciencia, Innovación y Universidades (Grant No. PGC2018-095862-BC21), the Comunidad de Madrid (Grant No. QUITEMAD+ S2013/ICE-2801), the "Centro de Excelencia Severo Ochoa" Programme (Grant No. SEV-2016-0597), and the CSIC Research Platform on Quantum Technologies PTI-001.
[1] R. Orús, Tensor networks for complex quantum systems, Nat. Rev. Phys. 1, 538 (2019).

[2] M. C. Bañuls, R. Blatt, J. Catani, A. Celi, J. I. Cirac, M. Dalmonte, L. Fallani, K. Jansen, M. Lewenstein, S. Montangero, C. A. Muschik, B. Reznik, E. Rico, L. Tagliacozzo, K. V. Acoleyen, F. Verstraete, U.-J. Wiese, M. Wingate, J. Zakrzewski, and P. Zoller, Simulating lattice gauge theories within quantum technologies, Europhys. J. D 74, 165 (2020).

[3] X. Chen, Z.-C. Gu, and X.-G. Wen, Classification of gapped symmetric phases in one-dimensional spin systems, Phys. Rev. B 83, 035107 (2011).

[4] N. Schuch, D. Perez-Garcia, and J. I. Cirac, Classifying quantum phases using matrix product states and projected entangled pair states, Phys. Rev. B 84, 165139 (2011).

[5] I. Affleck, T. Kennedy, E. H. Lieb, and H. Tasaki, Rigorous Results on Valence-Bond Ground States in Antiferromagnets, Phys. Rev. Lett. 59, 799 (1987).

[6] M. A. Levin and X. G. Wen, String-net condensation: A physical mechanism for topological phases, Phys. Rev. B 71, 045110 (2005).

[7] D. Perez-Garcia, M. M. Wolf, M. Sanz, F. Verstraete, and J. I. Cirac, String Order and Symmetries in Quantum Spin Lattices, Phys. Rev. Lett. 100, 167202 (2008).

[8] A. Fledderjohann, A. Klümper, and K.-H. Mütter, Diagrammatics for SU(2) invariant matrix product states, J. Phys. A: Math. Theor. 44, 475302 (2011).

[9] I. Kull, A. Molnar, E. Zohar, and J. I. Cirac, Classification of matrix product states with a local (gauge) symmetry, Ann. Phys. 386, 199 (2017).

[10] D. J. Williamson, N. Bultinck, and F. Verstraete, Symmetryenriched topological order in tensor networks: Defects, gauging and anyon condensation, arXiv:1711.07982.

[11] G. Mussardo, Statistical Field Theory: An Introduction to Exactly Solved Models in Statistical Physics (Oxford University Press, New York, 2010).

[12] V. Kalmeyer and R. B. Laughlin, Equivalence of the Resonating-Valence-Bond and Fractional Quantum Hall States, Phys. Rev. Lett. 59, 2095 (1987).
[13] X.-G. Wen, F. Wilczek, and A. Zee, Chiral spin states and superconductivity, Phys. Rev. B 39, 11413 (1989).

[14] M. Srednicki, Entropy and Area, Phys. Rev. Lett. 71, 666 (1993).

[15] J. Eisert, M. Cramer, and M. B. Plenio, Colloquium: Area laws for the entanglement entropy, Rev. Mod. Phys. 82, 277 (2010).

[16] G. Vidal, J. I. Latorre, E. Rico, and A. Kitaev, Entanglement in Quantum Critical Phenomena, Phys. Rev. Lett. 90, 227902 (2003).

[17] P. Calabrese and J. Cardy, Entanglement entropy and quantum field theory, J. Stat. Mech. (2004) P06002.

[18] A. Kapustin and L. Fidkowski, Local commuting projector Hamiltonians and the quantum Hall effect, Commun. Math. Phys. 373, 763 (2020).

[19] B. Béri and N. R. Cooper, Local Tensor Network for Strongly Correlated Projective States, Phys. Rev. Lett. 106, 156401 (2011).

[20] J. Dubail and N. Read, Tensor network trial states for chiral topological phases in two dimensions and a no-go theorem in any dimension, Phys. Rev. B 92, 205307 (2015).

[21] M. P. Zaletel and R. S. K. Mong, Exact matrix product states for quantum Hall wave functions, Phys. Rev. B 86, 245305 (2012).

[22] B. Estienne, Z. Papić, N. Regnault, and B. A. Bernevig, Matrix product states for trial quantum Hall states, Phys. Rev. B 87, 161112(R) (2013).

[23] B. Estienne, N. Regnault, and B. A. Bernevig, Fractional quantum Hall matrix product states for interacting conformal field theories, arXiv:1311.2936.

[24] J. I. Cirac and G. Sierra, Infinite matrix product states, conformal field theory, and the Haldane-Shastry model, Phys. Rev. B 81, 104431 (2010).

[25] G. Moore and N. Read, Nonabelions in the fractional quantum Hall effect, Nucl. Phys. B 360, 362 (1991).

[26] D. Jennings, C. Brockt, J. Haegeman, T. J. Osborne, and F. Verstraete, Continuum tensor network field states, path integral representations and spatial symmetries, New J. Phys. 17, 063039 (2015).

[27] A. Tilloy and J. I. Cirac, Continuous Tensor Network States for Quantum Fields, Phys. Rev. X 9, 021040 (2019). 
[28] F. D. M. Haldane, Exact Jastrow-Gutzwiller ResonatingValence-Bond Ground State of the Spin-1/2 Antiferromagnetic Heisenberg Chain with $1 / r^{2}$ Exchange, Phys. Rev. Lett. 60, 635 (1988).

[29] B. S. Shastry, Exact Solution of an $S=1 / 2$ Heisenberg Antiferromagnetic Chain with Long-Ranged Interactions, Phys. Rev. Lett. 60, 639 (1988).

[30] A. E. B. Nielsen, J. I. Cirac, and G. Sierra, Quantum spin Hamiltonians for the SU(2) $k$ WZW model, J. Stat. Mech. (2011) P11014.

[31] P. Di Francesco, P. Mathieu, and D. Sénéchal, Conformal Field Theory (Springer, New York, 1997).

[32] See Supplemental Material at http://link.aps.org/supplemental/ 10.1103/PhysRevB.103.155130 for computations of the functional and the sewing and closing conditions for the example in Sec. II and the functional for the example in Sec. III.
[33] R. Floreanini and R. Jackiw, Self-Dual Fields as ChargeDensity Solitons, Phys. Rev. Lett. 59, 1873 (1987).

[34] X. G. Wen, Gapless boundary excitations in the quantum Hall states and in the chiral spin states, Phys. Rev. B 43, 11025 (1991).

[35] A. E. B. Nielsen, J. I. Cirac, and G. Sierra, Laughlin Spin Liquid States on Lattices Obtained from Conformal Field Theory, Phys. Rev. Lett. 108, 257206 (2012).

[36] M. Green, J. H. Schwarz, and E. Witten, Superstring Theory, Vol. I (Cambridge University Press, Cambridge, U.K., 1987).

[37] J. Polchinsky, String Theory: An Introduction to the Bosonic String, Vol. I (Cambridge University Press, Cambridge, U.K., 1998).

[38] H.-H. Tu, A. E. B. Nielsen, J. I. Cirac, and G. Sierra, Lattice Laughlin states of bosons and fermions at filling fractions $1 / q$, New J. Phys. 16, 033025 (2014). 